\section{Beyond genomics}

F uture investments in genomics for genetic studies should have an appropriate epigenomic component if we are to prevail in understanding complex diseases with genetic and environmental causes. Our view is echoed by arguments presented by eminent epigeneticists at Nature's Horizon Symposium, 5-7 May 2005, at the Black Point Inn in Maine.

DNA sequencing has the best track record of delivering coordinating resources that have greatly reduced duplication of effort between labs. Good arguments have been made for further ambitious sequencing that will have the additional benefit of reducing per-unit costs to the range where new genetic experiments become possible. Newer uses of sequencing include SNP discovery, human genome diversity projects, microbial community sequencing, deep resequencing of candidate disease genes and, as discussed here, cancer genome projects.

As a rule, only rather moderate genetic risks have been associated with marker polymorphisms in studies of complex diseases, and in many cases, the causative variants have not been identified. We know of at least one published example of a SNP that conferred extreme allele-specific expression by an epigenetic mechanism (Nat. Genet. $36,497-501 ; 2004)$. It is also anticipated that environmental influences on complex diseases may be comparable to genetic influences. Many of these influences will be historical or difficult to quantify. Although comparing the sequences of cancer genomes with those of their host tissue may identify characteristic collections of point mutations consistent with extreme mutagenic exposures, the processes that contribute to an excess of point mutations have already yielded to analysis of the trans-acting mutator genes. In contrast, epigenetic marks, many of which await decoding, record the history of modifications imposed by the environment, often in ways that influence gene expression.

Although sequencing cancer genomes will identify point mutations in new cancer-associated genes, it is likely that the same oncogenes become activated and the same tumor-suppressor genes become silenced epigenetically. Better, the epigenomic approach applied to cancer progression can make sense of the patterns of coordinate deregulation and silencing of the several hundred well characterized cancer genes we already know. Although some drug targets may emerge from cancer genome projects, cancer epigenomics is already addressing the mechanisms of action of existing cancer therapies that use inhibitors of DNA methylase and histone deacetylase.

In competition with a finite deliverable, how can a case for more complex proposals best find traction? And which complementary functional experiments should be carried out along with each sequencing initiative to give most value to the information gained? In an ideal world, genomic and epigenomic projects would proceed in concord with one another and would both be designed to support investigations into function. This issue provides some examples of all three kinds of research. On page 590 Philip Stephens and colleagues report the largest complex disease genome sequencing study to date, covering somatic mutations in all the protein kinase coding exons, comprising $3 \%$ of the annotated coding regions of the human genome from several breast cancers and cancer cell lines. On page 645, Tsui-Ting Ching and colleagues present an epigenomic technology for identifying CpG methylation across the genome using BAC arrays.

Genomic and epigenomic researchers find their common ground in the determination of allele-specific expression. This phenomenon is currently laboriously established for particular combinations of alleles, in specific cells, under particular conditions. Establishing epigenetic markers of gene activity will greatly contribute to the detection of functional polymorphisms contributing to complex diseases. As shown in papers by Adam Raefski and Michael O'Neill and by William Davies and colleagues on pages 620 and 625, respectively, the epigenetic field has the most extensive experience in detecting allele-specific expression in the face of considerable complexity. In these studies, three $\mathrm{X}$-linked genes were found to be silenced by a paternal gametic imprint on the background of an X chromosome that had already undergone random $\mathrm{X}$ inactivation following an initial X-inactivating paternal gametic imprint.

Of course, not all the marks on chromosomes tell the same story, and we are not yet able to predict when DNA methylation and when histone modification predominantly influence gene expression. Still, on page 585 Peter Rugg-Gunn and colleagues establish that there is a consistent and mitotically stable pattern of epigenetic gene regulation of gametically imprinted genes in human embryonic stem cells in culture. This result provides important reassurance to those anticipating the development of stem cell therapy. Another urgent reason to study a wide range of stem cells is that the epigenetic mechanisms of stem cells in normal development are already providing plenty of clues to cancer. The same RNAi pathway that regulates heterochromatic gene silencing and histone methylation is essential for stem cell maintenance in fruit flies (Science 303; 669-672; 2004). The polycomb group protein Bmil is both essential for stem cell maintenance in mouse and overexpressed in human medulloblastomas (Nature $428,337-341 ; 2004$ ). So, it will be particularly interesting to compare stem cell epigenetic programs with those operating in the relentless self-renewal of cancer cells. 